\title{
Case report: identification of ERC1-RET fusion in a patient with pancreatic ductal adenocarcinoma
}

\author{
Jia Ma ${ }^{1}$, Baosheng Wang ${ }^{2}$, Erhong Meng ${ }^{3}$, Xiangpeng Meng ${ }^{2}$ \\ ${ }^{1}$ Department of Gastroenterology, The Fourth Affiliated Hospital of China Medical University, Shenyang, China; ${ }^{2}$ Pancreatic Endocrinology Ward, \\ Department of General Surgery, Shengjing Hospital of China Medical University, Shenyang, China; ${ }^{3}$ ChosenMed Technology (Beijing) Co. Ltd, \\ Beijing, China \\ Correspondence to: Xiangpeng Meng. Pancreatic Endocrinology Ward, Department of General Surgery, Shengjing Hospital of China Medical \\ University, No.36 Sanhao Street, Shenyang 110004, China. Email: mxpcmu@163.com.
}

\begin{abstract}
Pancreatic ductal adenocarcinoma (PDAC) is an aggressive malignancy. Currently, treatment strategies for PDAC are limited because its molecular characteristics have not yet been clarified. Different RET fusions have been reported in diverse solid tumors, especially in non-small cell lung cancer (NSCLC) and papillary thyroid carcinoma (PTC). Multikinase inhibitors (MKIs) such as cabozantinib, vandetanib and lenvatinib, as well as selective inhibitors of RET alterations like selpercatinib (LOXO-292) and pralsetinib (BLU-667) have been approved by the Food and Drug Administration (FDA) for patients with RET fusionpositive tumors, such as thyroid cancer, renal cell, NSCLC, and so on. However, few studies have been reported about the association between RET fusions and PDAC. ERC1-RET fusion is a rare rearrangement. To date, it has only been reported in lung cancer and thyroid cancer. Studies of ERC1-RET fusion in PDAC have not yet been explored. In this study, we reported an ERC1-RET fusion in a 60-year-old female patient with PDAC. To the best of our knowledge, this case was the first report about ERC1-RET fusion in a patient with PDAC. It is a pity that the patient refused targeted therapy for personal reasons. Our study has shed a new light on the companion diagnostics and targeted therapy for the patients with PDAC.
\end{abstract}

Keywords: Pancreatic ductal adenocarcinoma (PDAC); ERC1-RET fusion; RET inhibitors; case report

Submitted Jun 22, 2021. Accepted for publication Aug 17, 2021.

doi: $10.21037 /$ gs-21-469

View this article at: https://dx.doi.org/10.21037/gs-21-469

\section{Introduction}

Pancreatic ductal adenocarcinoma (PDAC) is the most common histological type of pancreatic cancer and accounts for approximately $90 \%$. It is a devastating cancer of the digestive system, which is hard to diagnose and treat (1). Approximately $80 \%$ of PDAC patients are diagnosed at an advanced stage (2). Therefore, it is urgent to seek out precision therapy based on the genetic alterations of PDAC.

With the increasing application of next-generation sequencing (NGS) in tumors, more and more therapeutic targets have been found in PDAC. For example, patients with $\mathrm{PDAC}$ who harbor variations in homologous recombination genes such as $B R C A 1, B R C A 2, P A L B 2$, and so on, usually respond well to poly [adenosine diphosphate (ADP)-ribose] polymerase inhibitors and cisplatin (3). In addition, patients with mismatch repair gene mutations can often gain clinical benefit from immune checkpoint inhibitors (4). Moreover, patients harboring NTRK gene fusion are sensitive to larotrectinib and entrectinib (5). However, these "actionable" genetic mutations only account for a fraction of PDAC. Thus, there is an urgent need to identify more molecular targets.

The proto-oncogene RET encodes a membrane receptor 

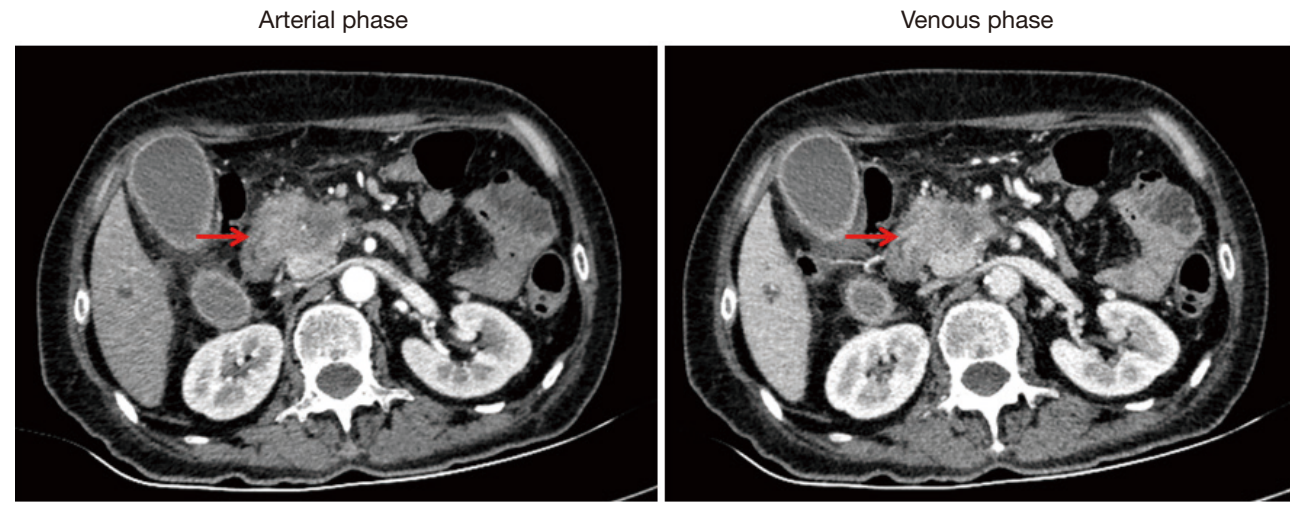

Figure $1 \mathrm{CT}$ images of the patient. Arterial and venous phase CT images in the pancreatic head and neck (red arrow refers to the spaceoccupying lesion). CT, computed tomography.

tyrosine kinase (6). Under normal conditions, the RET gene plays a crucial role in kidney and nervous system development (7-10). When aberrantly activated, RET gene can act as an oncogenic driver in a variety of cancer types. The RET gene is abnormally activated mainly through point mutations and chromosomal rearrangements. The latter will generate fusion proteins including the kinase domain of RET. Apart from those 2 ways, the RET gene can also be activated aberrantly by over expression of wild-type RET (10).

Studies have shown that RET fusions retaining kinase domain are oncogenic drivers in $5-10 \%$ of sporadic papillary thyroid carcinoma (PTC) and $1-2 \%$ of non-small cell lung cancer (NSCLC) (11). Besides, RET gene fusions are also drivers in other tumors such as colorectal cancer, breast cancer, PDAC, and so on, in which they account for $<1 \%$ of the total cases of each type $(6,12)$. Specifically, according to the investigation by Kato et al, the occurrence rate of RET fusion in PDAC is $0.6 \%$ (6).

To date, it has been reported that there are more than 35 partners of RET gene fusions. In PTC, the most frequent partners are the CCDC6 and NCOA4 genes. In NSCLC, the most common partners are KIF5B, CCDC6, and NCOA4 genes $(10,13,14)$.

The ELKS/Rab6-interacting/CAST family member 1 (ERC1) gene is located at $12 \mathrm{p} 13.3$, and is ubiquitously expressed (15). Previous studies have reported that its product ERC1/ELKS scaffold protein could promote migration and invasion of tumor cells $(16,17)$.

The ERC1-RET fusion is a rare rearrangement. To date, it has only been reported in lung cancer and thyroid carcinoma $(18,19)$. The study of ERC1-RET fusion in PDAC has not yet been explored.

In this study, an ERC1-RET fusion was detected through NGS and verified by Sanger sequencing in a patient with PDAC. To the best of our knowledge, this case was the first report about ERC1-RET fusion in a patient with PDAC. We present the following case in accordance with the CARE reporting checklist (available at https://dx.doi.org/10.21037/ gs-21-469).

\section{Case presentation}

The study conformed to the provisions of the Declaration of Helsinki (as revised in 2013). It was approved by the Ethics Committee of Shengjing Hospital of China Medical University. Written informed consent was obtained from the patient for publication of this case report and accompanying images. A copy of the written consent is available for review by the editorial office of this journal. A 60-year-old female patient was referred to our hospital on 25 August 2020 due to her skin and sclera having turned yellow for 1 month. She had no abdominal pain, distension, vomiting, or dizziness, but had experienced occasional nausea. In addition, the patient complained of loss of appetite and compromised defecation with claycolored stool. She had lost about $15 \mathrm{~kg}$ over the past month. Abdominal enhanced computed tomography (CT) showed a space-occupying lesion of the pancreatic head and neck (Figure 1). Enhanced magnetic resonance imaging (MRI) 


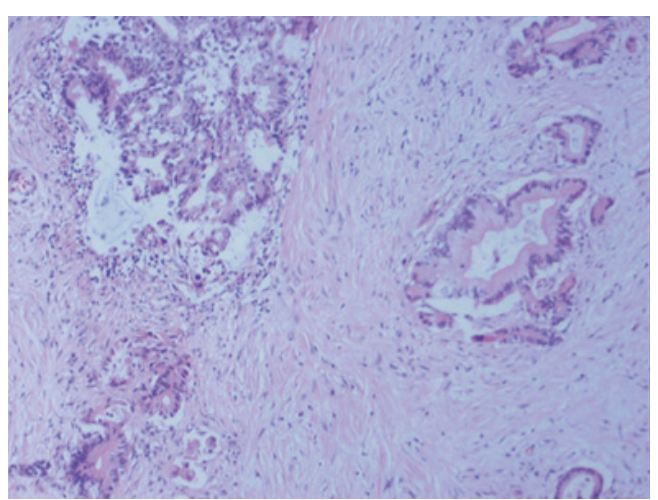

Figure 2 Pathological findings of the patient. Pathological findings confirmed the space-occupying lesion was PDAC $(\mathrm{HE}, \times 200)$. PDAC, pancreatic ductal adenocarcinoma.

indicated that the space-occupying lesion was liable to be malignant. No systematic diagnosis and treatment was given to the patient at that time. On 15 September 2020, the patient underwent the Whipple operation. Her pathological diagnosis was moderately to highly differentiated PDAC (pT3N0M0, Figure 2).

In order to seek personalized treatment options, paraffinembedded samples from surgical resection of PDAC in the patient were subjected to NGS through a 599-gene panel [ChosenOne599, ChosenMed Technology (Beijing) Co. Ltd, Beijing, China] on 15 September 2020. The NGS data analysis revealed that the patient harbored an ERC1-RET fusion, and the allele frequency was $5.07 \%$. This fusion included exons 1-16 of ERC1 on chromosome 12 and exons 12-20 of RET on chromosome 10, which retained the complete kinase domain of RET (Figure $3 A, 3 B$ and Table 1).

Sequencing reads of ERC1-RET fusion were shown by the Integrative Genome Viewer (IGV) in Figure $3 A$. Somatic mutations in the patient are demonstrated in Table 1.

Sanger sequencing was carried out to verify the ERC1RET fusion using the same tumor samples from the patient (Tsingke Biotechnology Co., Ltd, Tianjin, China). The results of Sanger sequencing confirmed that the patient harbored the fusion (Figure 3C).

The timeline in the patient is shown in Figure 4.

\section{Discussion}

In this case, the chimeric ERC1-RET protein contains complete kinase domain encoded by RET exons $12-20$ and coiled-coil domain encoded by ERC1 exons 1-16, which might activate the downstream signaling pathways and drive oncogenesis (12).

To the best of our knowledge, this case was the first study of ERC1-RET fusion in a patient with PDAC.

New targeted drugs for RET fusion are emerging in succession. In the past decade, the FDA has approved multikinase inhibitors (MKIs) with ancillary RET inhibitor activity such as cabozantinib and vandetanib to treat medullary thyroid cancer, cabozantinib to treat renal cell cancer and hepatocellular carcinoma, lenvatinib to treat thyroid cancer and renal cell cancer, and so on. Recently, with FDA-approved potent and highly selective RET inhibitors selpercatinib (LOXO-292) and pralsetinib (BLU-667), precision treatment of RET-positive NSCLC and thyroid cancer has reached a new stage. Moreover, previous studies have indicated that therapeutic efficacy of these inhibitors might depend on RET fusion partners and variants. For instance, Drilon et al. evaluated the clinical response of patients with lung cancer who received treatment with cabozantinib. Their results confirmed that responses occurred in $20 \%$ of patients (3/15) harboring KIF5B-RET fusion; however, no clinical benefit was observed in patients harboring CCDC6-RET or ERC1-RET fusion (18).

Although RET fusion is a rare mutation in PDAC, the number of patients with PDAC in China is much higher than that in other countries due to the large population. It is suggested that patients with PDAC should be tested by NGS if possible. If RET fusion is detected, patients will have more treatment options and more chances of survival.

Thus, although no targeted drug has been approved for RET fusion-positive PDAC, PDAC will be diagnosed and treated more accurately in the future based on the increasing application of NGS detection technology.

Our study had presented a new molecular target for the treatment of PDAC. The limitation of the study was that the participant refused to receive inhibitors of RET due to personal reasons.

\section{Conclusions}

In summary, this case firstly reported an ERC1-RET fusion in a patient with PDAC. Our study discovered a novel 

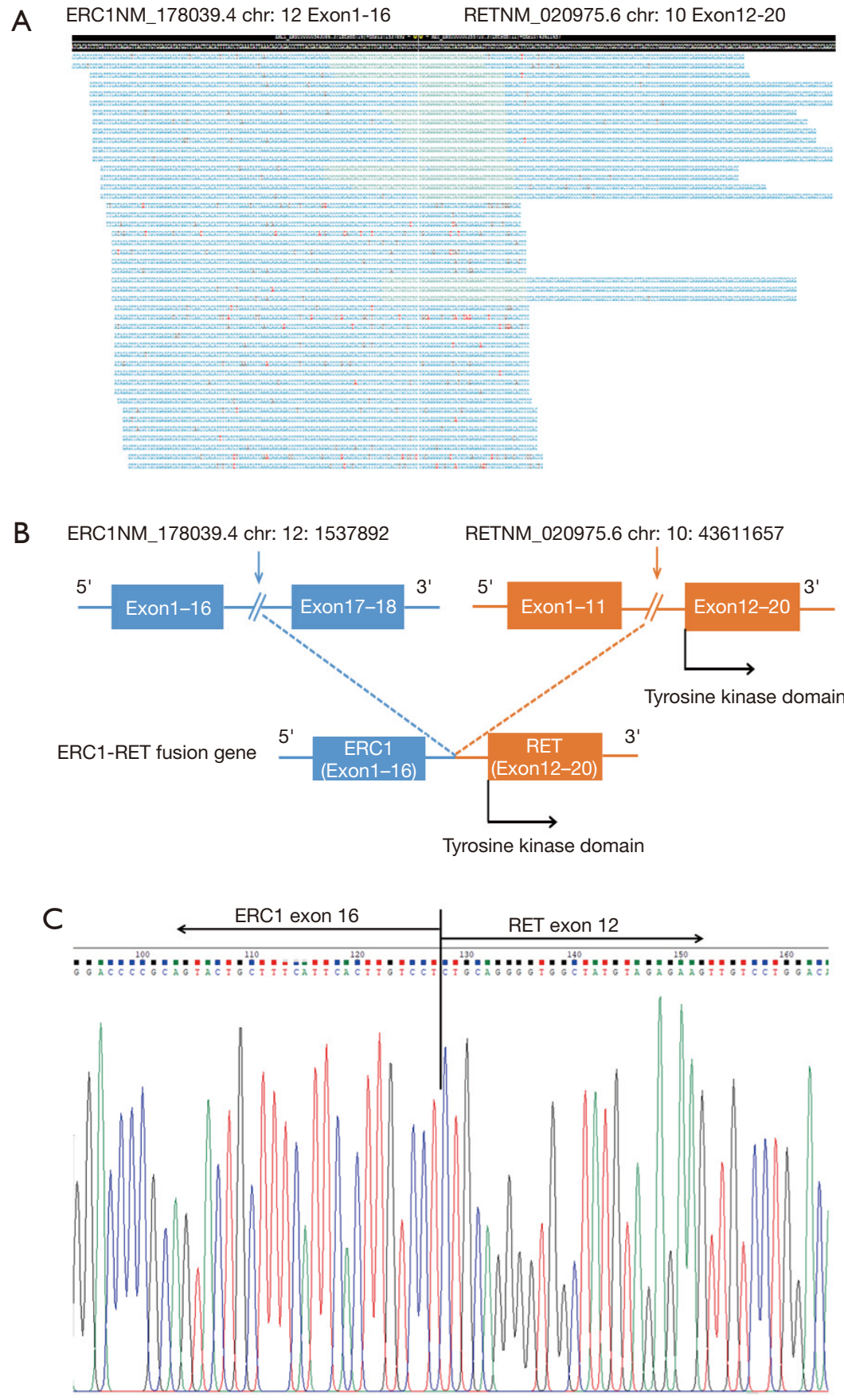

Figure 3 ERC1-RET fusion was identified by NGS and verified through Sanger sequencing. (A) Sequencing reads of ERC1-RET fusion were shown by IGV; (B) Schematic diagram of ERC1-RET fusion; (C) ERC1-RET fusion was verified by Sanger sequencing. NGS, nextgeneration sequencing; IGV, Integrative Genomics Viewer. 
Table 1 Somatic variations in the patient.

\begin{tabular}{lccccc}
\hline Gene & Transcript & Exon & Nucleotide change & Alteration & Mutant allele frequency/copy number \\
\hline PIK3CA & NM_006218 & 2 & c.317G>T & p.G106V & $31.30 \%$ \\
PIK3CA & NM_006218 & 2 & c.328_330del & p.E110del & $4.40 \%$ \\
TP53 & NM_000546 & 7 & c.742C>T & p.R248W & $48.40 \%$ \\
ARID5B & NM_032199 & 6 & c.982C>A & p.L328I & $2.00 \%$ \\
INPP4A & NM_001134224 & 20 & c.2104T>C & p.F702L & $28.70 \%$ \\
KMT2D & NM_003482 & 10 & c.1930A>C & p.M644L & $4 \%$ \\
SUZ12 & NM_015355 & 1 & c.29G>A & p.G10E & $30.70 \%$ \\
CDKN2A & - & - & Loss & - & 1 \\
CDKN2B & - & - & Loss & - & 1 \\
ERC1-RET & - & - & ERC1(intron 16)-RET(intron 11) & $5.07 \%$ \\
\hline
\end{tabular}

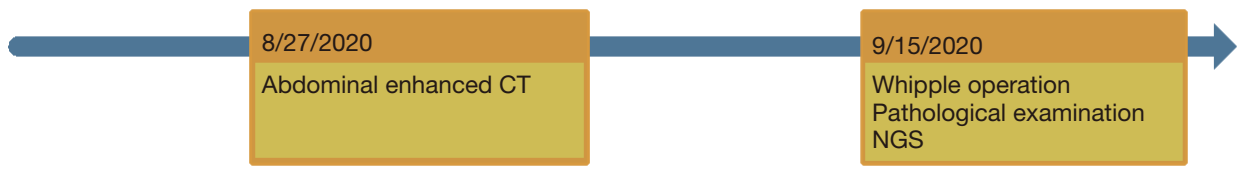

Figure 4 Timeline of the patient.

molecular target and has provided clues for the application of RET protein-tyrosine kinase inhibitors in patients with PDAC.

\section{Acknowledgments}

The authors wish to thank the patient and all personnel for participating in this study.

Funding: None.

\section{Footnote}

Reporting Checklist: The authors have completed the CARE reporting checklist. Available at https://dx.doi. org/10.21037/gs-21-469

Conflicts of Interest: All authors have completed the ICMJE uniform disclosure form (available at https:// dx.doi.org/10.21037/gs-21-469). EM is an employee of ChosenMed Technology. The other authors have no conflicts of interest to declare.

Ethical Statement: The authors are accountable for all aspects of the work in ensuring that questions related to the accuracy or integrity of any part of the work are appropriately investigated and resolved. The study conformed to the provisions of the Declaration of Helsinki (as revised in 2013). It was approved by the Ethics Committee of Shengjing Hospital of China Medical University. Written informed consent was obtained from the patient for publication of this case report and accompanying images. A copy of the written consent is available for review by the editorial office of this journal.

Open Access Statement: This is an Open Access article distributed in accordance with the Creative Commons Attribution-NonCommercial-NoDerivs 4.0 International License (CC BY-NC-ND 4.0), which permits the noncommercial replication and distribution of the article with the strict proviso that no changes or edits are made and the original work is properly cited (including links to both the formal publication through the relevant DOI and the license). See: https://creativecommons.org/licenses/by-nc-nd/4.0/.

\section{References}

1. Torre LA, Bray F, Siegel RL, et al. Global cancer statistics, 2012. CA Cancer J Clin 2015;65:87-108. 
2. Nagaria TS, Wang H. Modification of the 8th AJCC staging system of pancreatic ductal adenocarcinoma. Hepatobiliary Surg Nutr 2020;9:95-7.

3. Lowery MA, Kelsen DP, Stadler ZK, et al. An emerging entity: pancreatic adenocarcinoma associated with a known BRCA mutation: clinical descriptors, treatment implications, and future directions. Oncologist 2011;16:1397-402.

4. Le DT, Uram JN, Wang H, et al. PD-1 Blockade in Tumors with Mismatch-Repair Deficiency. N Engl J Med 2015;372:2509-20.

5. O'Reilly EM, Hechtman JF. Tumour response to TRK inhibition in a patient with pancreatic adenocarcinoma harbouring an NTRK gene fusion. Ann Oncol 2019;30 Suppl 8:viii36-viii40.

6. Kato S, Subbiah V, Marchlik E, et al. RET Aberrations in Diverse Cancers: Next-Generation Sequencing of 4,871 Patients. Clin Cancer Res 2017;23:1988-97.

7. Nakamura T, Ishizaka $Y$, Nagao $M$, et al. Expression of the ret proto-oncogene product in human normal and neoplastic tissues of neural crest origin. J Pathol 1994;172:255-60.

8. Wells SA Jr, Santoro M. Targeting the RET pathway in thyroid cancer. Clin Cancer Res 2009;15:7119-23.

9. Takahashi M, Buma Y, Iwamoto T, et al. Cloning and expression of the ret proto-oncogene encoding a tyrosine kinase with two potential transmembrane domains. Oncogene 1988;3:571-8.

10. Subbiah V, Yang D, Velcheti V, et al. State-of-the-Art Strategies for Targeting RET-Dependent Cancers. J Clin Oncol 2020;38:1209-21.

Cite this article as: Ma J, Wang B, Meng E, Meng X. Case report: identification of ERC1-RET fusion in a patient with pancreatic ductal adenocarcinoma. Gland Surg 2021;10(9):28742879. doi: $10.21037 /$ gs-21-469
11. Kohno T, Tabata J, Nakaoku T. REToma: a cancer subtype with a shared driver oncogene. Carcinogenesis 2020;41:123-9.

12. Wiesner T, He J, Yelensky R, et al. Kinase fusions are frequent in Spitz tumours and spitzoid melanomas. Nat Commun 2014;5:3116.

13. Santoro M, Carlomagno F. Central role of RET in thyroid cancer. Cold Spring Harb Perspect Biol 2013;5:a009233.

14. Subbiah V, Cote GJ. Advances in Targeting RETDependent Cancers. Cancer Discov 2020;10:498-505.

15. Gorello P, La Starza R, Brandimarte L, et al. A PDGFRBpositive acute myeloid malignancy with a new $\mathrm{t}(5 ; 12)$ (q33;p13.3) involving the ERC1 gene. Leukemia 2008;22:216-8.

16. Astro V, Asperti C, Cangi MG, et al. Liprin- $\alpha 1$ regulates breast cancer cell invasion by affecting cell motility, invadopodia and extracellular matrix degradation. Oncogene 2011;30:1841-9.

17. Astro V, Chiaretti S, Magistrati E, et al. Liprin- $\alpha 1$, ERC1 and LL5 define polarized and dynamic structures that are implicated in cell migration. J Cell Sci 2014;127:3862-76.

18. Drilon A, Rekhtman N, Arcila M, et al. Cabozantinib in patients with advanced RET-rearranged non-small-cell lung cancer: an open-label, single-centre, phase 2, singlearm trial. Lancet Oncol 2016;17:1653-60.

19. Chu YH, Wirth LJ, Farahani AA, et al. Clinicopathologic features of kinase fusion-related thyroid carcinomas: an integrative analysis with molecular characterization. Mod Pathol 2020;33:2458-72.

(English Language Editor: J. Jones) 